\title{
Cidades inteligentes como nova prática para o gerenciamento dos serviços e infraestruturas urbanos: a experiência da cidade de Porto Alegre
}

\author{
Smart cities as a new practice for urban services and infrastructure \\ management: the experience of Porto Alegre
}

Marcos Cesar Weiss ${ }^{[0]}$, Roberto Carlos Bernardes ${ }^{[0]}$, Flavia Luciane Consoni ${ }^{[b]}$

[a] Centro Universitário da Faculdade de Engenharia Industrial (FEI), São Paulo, SP, Brasil

[b] Universidade Estadual de Campinas (Unicamp), Campinas, SP, Brasil

\section{Resumo}

Este artigo tem por objetivo discutir a experiência da cidade de Porto Alegre relativamente à materialização do conceito de cidade inteligente. Para tanto, utilizou-se uma abordagem metodológica de caráter qualitativo e exploratório, baseada em estudo de caso, com coleta de dados em fontes primárias e secundárias realizada em 2013. Os resultados mostram que a implementação de inovações em tecnologias da informação e comunicação (TICs) na prestação dos serviços públicos proporcionou maior eficiência nas atividades que envolvem a gestão da cidade, com importantes resultados em favor dos atores que ali se inserem. Pretendeu-se com este trabalho contribuir com a agenda de pesquisas e discussões sobre a gestão dos espaços urbanos, trazendo o conceito de cidade inteligente como uma prática viabilizadora do desenvolvimento sustentável das cidades brasileiras.

Palavras-chave: Cidades inteligentes. Cidades digitais. Gestão da inovação tecnológica das cidades.

\section{Abstract}

This paper aims to discuss the experience of Porto Alegre regarding to the smart city concept implementation. Therefore, we used a study-based qualitative and exploratory methodological approach with data collection performed in primary and secondary sources in 2013. The results show that the implementation of information and communication technologies (ICT) innovations to deliver public services provided greater efficiency in activities involving the city management, with significant results to the actors who work and live there. This work sought to contribute to the research agenda and discussions on urban space management, bringing the concept of smart city as a pragmatic enabler for sustainable development for Brazilian cities.

Keywords: Smart cities. Digital cities. Technological urban innovation management.

MCW é doutorando em Administração pelo Centro Universitário da FEI, e-mail: mw@marcosweiss.com.br

RCB é Doutor em Sociologia pela USP, professor Adjunto do Programa de Pós-graduação em Administração e Pesquisador do Centro Universitário da FEI, e-mail: bernardes@fei.edu.br

FLC é doutora em Política Científica e Tecnológica pela Unicamp, com pós-doutorado em Sociologia pela USP, professora de Mestrado e Doutorado em Política Científica e Tecnológica na Unicamp, e-mail: flavia@ige.unicamp.br 


\section{Introdução}

Projeções da Organização das Nações Unidas (ONU) indicam que a população mundial crescerá em mais de 2 bilhões de pessoas nos próximos 40 anos, ultrapassando o patamar de 9 bilhões de habitantes, e mais de 65\% deles viverão em cidades (ONU, 2012).

As perspectivas globais sobre a dramática aglomeração urbana representam importantes desafios para a gestão pública. Tais desafios, decorrentes da pressão por efetividade no atendimento às demandas sociais, exigem novas abordagens para seu planejamento, projeto, financiamento, execução e operação (Boyko et al., 2006; Harrison \& Donnelly, 2011; Rasoolimanesh et al., 2011). O cenário torna-se mais desafiador quando consideradas as restrições de natureza legal-institucional e econômica para a destinação de recursos públicos e a competição cada vez mais agressiva entre as cidades para a atração de investimentos, visto que as receitas dos governos geralmente chegam mais tarde do que as demandas ou são equivocadamente destinadas (Nam \& Pardo, 2011a; Cadena et al., 2012).

Nesse cenário, as cidades passam a ser concebidas como espaços geoeconômicos críticos para o acesso aos fluxos globais de conhecimento, às redes transnacionais para a geração de riqueza e criação de valor, por meio de ecossistemas institucionais técnico-científicos públicos, privados e de arquiteturas empresariais propícias ao fomento de soluções inovadoras e inclusivas (Sassen, 1998; Hammer et al., 2011; Castells, 2012).

Nessa perspectiva, o conceito de cidade inteligente surge como uma nova dimensão da gestão pública para o enfrentamento desses desafios.

Embora se sustente em infraestruturas digitais, a cidade inteligente depende do desenvolvimento contínuo da capacidade de aprendizagem para a inovação e replicação nos processos de gestão da dinâmica urbana (Hernández-Muñoz et al., 2011; Komninos et al., 2011). Ela utiliza as capacidades da cidade digital para implementar sistemas de informações que melhorem a disponibilidade e a qualidade das infraestruturas e serviços públicos, incrementando sua capacidade de crescimento e estimulando a inovação e o desenvolvimento sustentável. Isso significa que a cidade digital não é necessariamente inteligente, mas a cidade inteligente tem, obrigatoriamente, componentes digitais (Allwinkle \& Cruickshank, 2011; Dutta, 2011; Nam \& Pardo, 2011b).
Essas reflexões iniciais remetem à pergunta de pesquisa que orienta o desenvolvimento deste artigo: como o conceito de cidades inteligentes vem sendo aplicado pelas cidades brasileiras com vistas a um novo modelo de gestão pública?

Para responder à pergunta de pesquisa, este trabalho apresenta e analisa a iniciativa de implementação de cidade inteligente em Porto Alegre (POA), reconhecida, tanto nacional quanto internacionalmente (Nam \& Pardo, 2011a), como uma das principais metrópoles brasileiras pioneiras na implementação de projetos associados ao conceito de cidade inteligente. Nesse sentido, busca-se compreender como tem ocorrido a materialização do conceito nesta localidade, superando o estágio de cidade digital. 0 foco recai na forma como as tecnologias da informação e comunicação (TICs) têm avançado na promoção de uma gestão eficiente das infraestruturas e serviços urbanos que contemplem os interesses e as demandas dos diversos atores.

Pretende-se contribuir com a arena de debates acerca das questões que envolvem o futuro das cidades, procurando refletir sobre como as inovações em TICs podem possibilitar aos governos melhor enfrentamento aos desafios e aproveitamento de oportunidades para melhoria da qualidade de vida nesses ambientes.

0 artigo está organizado em cinco seções. A seção seguinte, orientada pelo conceito de cidades inteligentes, contextualiza as cidades no atual cenário global e aborda seu papel como polo para a geração de inovações e ponto de partida para o desenvolvimento sustentável. A terceira seção apresenta os procedimentos metodológicos e, na quarta seção, são apresentados os resultados da pesquisa, iniciando-se pela caracterização do ambiente de pesquisa e seguindo-se a narrativa das ações presentes e dos planos de seguimento da iniciativa. A quinta seção encerra o trabalho, com as considerações finais e proposições para futuros estudos.

\section{As cidades do futuro: cidades inteligentes}

Os debates sobre o papel das cidades na economia global têm se intensificado, particularmente quando se observa a intensa concentração das pessoas nas cidades (Sassen, 1998).

Para diversos autores, é de fundamental importância relacionar as tendências socioeconômicas com 
as características das cidades (Friedmann, 1986; Brenner, 1998; Bollier, 1998; Scott et al., 2001; Parkinson et al., 2004; Sassen, 2005; Pastor, Lester \& Scoggins, 2009; Cohen, 2011). Para esses autores, as cidades funcionam como espaços-chave da economia global, onde se observam os efeitos da globalização: forte acúmulo e fluxo de capital, desindustrialização, expansão e concentração espacial de setores manufatureiro e de serviços, segmentação do mercado de trabalho, conflitos étnicos e de classes, polarização socioespacial.

O cenário, entretanto, mostra que a intensa urbanização traz perdas de funcionalidades básicas, afetando significativamente a qualidade de vida da população: deficiências na gestão de resíduos; escassez, desperdícios e má gestão dos recursos naturais; restrições nos sistemas de saúde, educação e segurança pública; limitações nos sistemas de mobilidade urbana e de transportes; obsolescência e encurtamento do ciclo de vida das infraestruturas públicas (Toppeta, 2010; Batagan, 2011). Para Gupta (2002), Johnson (2008), Toppeta (2010), Washburn \& Sindhu (2010), Nam \& Pardo (2011a), Batagan (2011), Dodgson \& Gann (2011) e Dutta (2011), essas restrições podem ser enfrentadas com o aproveitamento adequado das capacidades atuais e futuras, melhorando a eficiência e reinventando a organização das cidades, tendo as TICs como viabilizadoras de um sistema nervoso para e de cidades inteligentes.

Como as cidades estão mais interconectadas e instrumentalizadas (Harrison \& Donnelly, 2011), o seu sucesso se direciona sobre dois eixos principais. Primeiro, o gerenciamento dos recursos a partir de uma perspectiva sustentável para que se tornem ambientalmente adequadas e atraentes para os atores, implementando um tipo de gestão mais inovador, permitindo que sejam mais preditivas e integrando o conjunto dos seus ativos de forma ágil e a custos aceitáveis (Al-hader \& Rodzi, 2009; Washburn \& Sindhu, 2010; Harrison \& Donnelly, 2011; Wolfram, 2012; Toppeta, 2010). Segundo, pela criação de um ambiente atrativo do ponto de vista econômico-social, onde os atores possam interagir sem restrições que mereçam intervenções drásticas, imprimindo maior eficiência e reformulando a organização da dinâmica urbana. As TICs seriam, portanto, as viabilizadoras de um modelo capaz de implementar maior inteligência nestas cidades (Storper, 1997; Gupta, 2002; Johnson, 2008; Eger, 2009; Dodgson \& Gann, 2011; Cadena et al., 2012).
Ao longo do tempo, muitas definições foram somadas ao cenário dos estudos para a criação de novas formas e tecnologias para o gerenciamento das cidades, resultando no conceito de cidade inteligente.

Antes, porém, de se adentrar ao conceito de cidade inteligente, é imperativo marcar sua distinção em relação ao conceito de cidade digital.

A cidade digital é caracterizada primordialmente pela capacidade de implementação de tecnologias de comunicação, promovendo o acesso amplo a ferramentas, conteúdos e sistemas de gestão, de forma a atender às necessidades do poder público e seus servidores, dos cidadãos e das organizações (Komninos, 2002; Yovanof \& Hazapis, 2009). Já a cidade inteligente emerge da cidade digital. A visão de inteligência das cidades vem da convergência entre a sociedade do conhecimento - onde a informação e a criatividade têm grande ênfase e que considera os capitais humano e social como seus mais valiosos ativos (Castells, 2012) - e a cidade digital - que faz extensivo uso de sistemas de telecomunicações e recursos da internet como meio para transformar significativamente as formas de relacionamento e de vida (Kanter \& Litow, 2009; Coelho, 2010; Nam \& Pardo, 2011b).

Para Komninos \& Sefertzi (2009), as iniciativas para cidades inteligentes focalizam o uso das TICs para transformar a vida e o trabalho dentro de uma região, de forma significativa e fundamental, mais do que de forma incremental, explorando os recursos da cidade digital de maneira inovadora e colaborativa. Nesse sentido, a cidade digital não é necessariamente inteligente, mas a cidade inteligente tem, obrigatoriamente, componentes digitais (Allwinkle \& Cruickshank, 2011; Dutta, 2011; Nam \& Pardo, 2011b).

Ao longo dos últimos anos, muitos autores ofereceram definições para o termo cidades inteligentes, como apresentado no Quadro 1.

Outros autores, como Schaffers et al. (2011), Hernández-Muñoz et al. (2011), Chourabi et al. (2012) e Cadena et al. (2012), alinham-se à conceituação proposta por Dutta (2011).

A abordagem de cidades inteligentes inclui tecnologias que promovem maior eficiência energética e otimização na produção de bens e serviços; sistemas inteligentes para o monitoramento e gerenciamento das infraestruturas urbanas e antecipação a acidentes naturais; soluções de colaboração e redes sociais; sistemas integrados para a gestão de ativos; sistemas especializados de 
Quadro 1 - Definiç,ões de cidades inteligentes

\begin{tabular}{|c|c|}
\hline Autor & Definição \\
\hline Hall et al. (2000) & $\begin{array}{l}\text { São aquelas que monitoram e integram as condições de operaccões de todas as infraestruturas críticas da cidade, atuando de forma } \\
\text { preventiva para a continuidade de suas atividades fundamentais. }\end{array}$ \\
\hline Kanter \& Litow (2009) & $\begin{array}{l}\text { São aquelas capazes de conectar de forma inovadora as infraestruturas físicas e de TICs, eficiente e eficazmente, convergindo os } \\
\text { aspectos organizacionais, normativos, sociais e tecnológicos a fim de melhorar as condições de sustentabilidade e de qualidade vida da } \\
\text { população. }\end{array}$ \\
\hline Toppeta (2010) & $\begin{array}{l}\text { São aquelas que combinam as facilidades das TICS e da Web } 2.0 \text { com os esforços organizacionais, de design e planejamento, para } \\
\text { desmaterializar e acelerar os processos burocráticos, ajudando a identificar e implementar soluções inovadoras para o gerenciamento da } \\
\text { complexidade das cidades. }\end{array}$ \\
\hline Giffinger \& Gudrun (2010) & $\begin{array}{l}\text { São aquelas que bem realizam a visão de futuro em várias vertentes - economia, pessoas, governança, mobilidade, meio ambiente e } \\
\text { qualidade de vida - e são construídas sobre a combinação inteligente de atitudes decisivas, independentes e conscientes dos atores que } \\
\text { nelas atuam. }\end{array}$ \\
\hline Washburn \& Sindhu (2010) & $\begin{array}{l}\text { São aquelas que usam tecnologias de smart computing para tornar os componentes das infraestruturas e serviçcos críticos - os quais } \\
\text { incluem a administração da cidade, educação, assistência à saúde, segurança pública, edifícios, transportes e utilities - mais inteligentes, } \\
\text { interconectados e eficientes. }\end{array}$ \\
\hline Dutta (2011) & $\begin{array}{l}\text { São aquelas que têm foco em um modelo particularizado, com visão moderna do desenvolvimento urbano e que reconhecem a } \\
\text { crescente importância das tecnologias da informação e comunicação no direcionamento da competitividade econômica, sustentabilidade } \\
\text { ambiental e qualidade de vida geral; esse conceito vai além dos aspectos puramente técnicos que caracterizam as cidades como cidades } \\
\text { digitais. }\end{array}$ \\
\hline Harrison \& Donnelly (2011) & $\begin{array}{l}\text { São aquelas que fazem uso sistemático das TICS para promover a eficiência no planejamento, execução e manutenção dos serviços e } \\
\text { infraestruturas urbanos, no melhor interesse dos atores que atuam nestas cidades. }\end{array}$ \\
\hline Nam \& Pardo (2011a) & $\begin{array}{l}\text { São aquelas que têm por objetivo a melhoria na qualidade dos serviços aos cidadãos e que o estabelecimento de sistemas integrados } \\
\text { baseados em TICS não é um fim em si, mas mecanismos por meio dos quais os serviçcos são fornecidos e as informações são comparti- } \\
\text { lhadas. }\end{array}$ \\
\hline
\end{tabular}

Fonte: Elaborado pelos autores (2014), com base na bibliografia.

atenção à saúde e educação que permitem a interação com os atores por intermédio da internet; sistemas, métodos e práticas para o gerenciamento integrado de serviços de qualquer natureza; sistemas para o tratamento de grandes volumes de dados estruturados e não estruturados; sistemas de georreferenciamento; aplicações inteligentes embarcadas em toda sorte de bens; tecnologias de identificação por radiofrequência e etiquetas digitais colocadas em produtos e cargas, otimizando os processos logísticos e as transações comerciais; sensores e sistemas de inteligência artificial que percebem e respondem rapidamente a eventos ocorridos no mundo físico, desencadeando processos digitais que passam a ter consequências cada vez mais imediatas e significativas no mundo, conectando pessoas, empresas e poder público a qualquer tempo e em qualquer lugar (Mitchell, 2007; Webber \& Wallace, 2009; Dirks et al., 2010; Prattipati, 2010; Allwinkle \& Cruickshank, 2011; Wolfram, 2012).

Consideradas as definições e os estudos realizados pelos autores citados neste trabalho, propõe-se uma caracterização evolutiva da cidade digital à cidade inteligente, como na Figura 1, partindo da implementação de um computador isolado (stand alone $P C$ ) até sua mais recente perspectiva tecnológica - a internet das coisas (Internet of Things).

Todas as possibilidades presentes e futuras proporcionadas pelas TICs contribuem para a redução nas emissões de gases de efeito estufa, porquanto viabilizam maior eficiência no uso dos recursos materiais, técnicos e humanos. Elas podem implementar novos canais de comunicação entre os atores, melhorar a eficiência, a transparência e a democratização no acesso a informações, contribuindo para que melhores escolhas sejam feitas e melhores decisões sejam tomadas.

As aplicações de TICs para a criação de cidades inteligentes são inúmeras e podem ser disponibilizadas e adaptadas às necessidades e características de cada cidade, como proposto no framework de sistemas integrados para as cidades inteligentes na Figura 2.

Criar cidades inteligentes não se trata de uma revolução, de um conceito tecnológico ou de um fenômeno localizado particularmente. Trata-se, ao contrário, de 


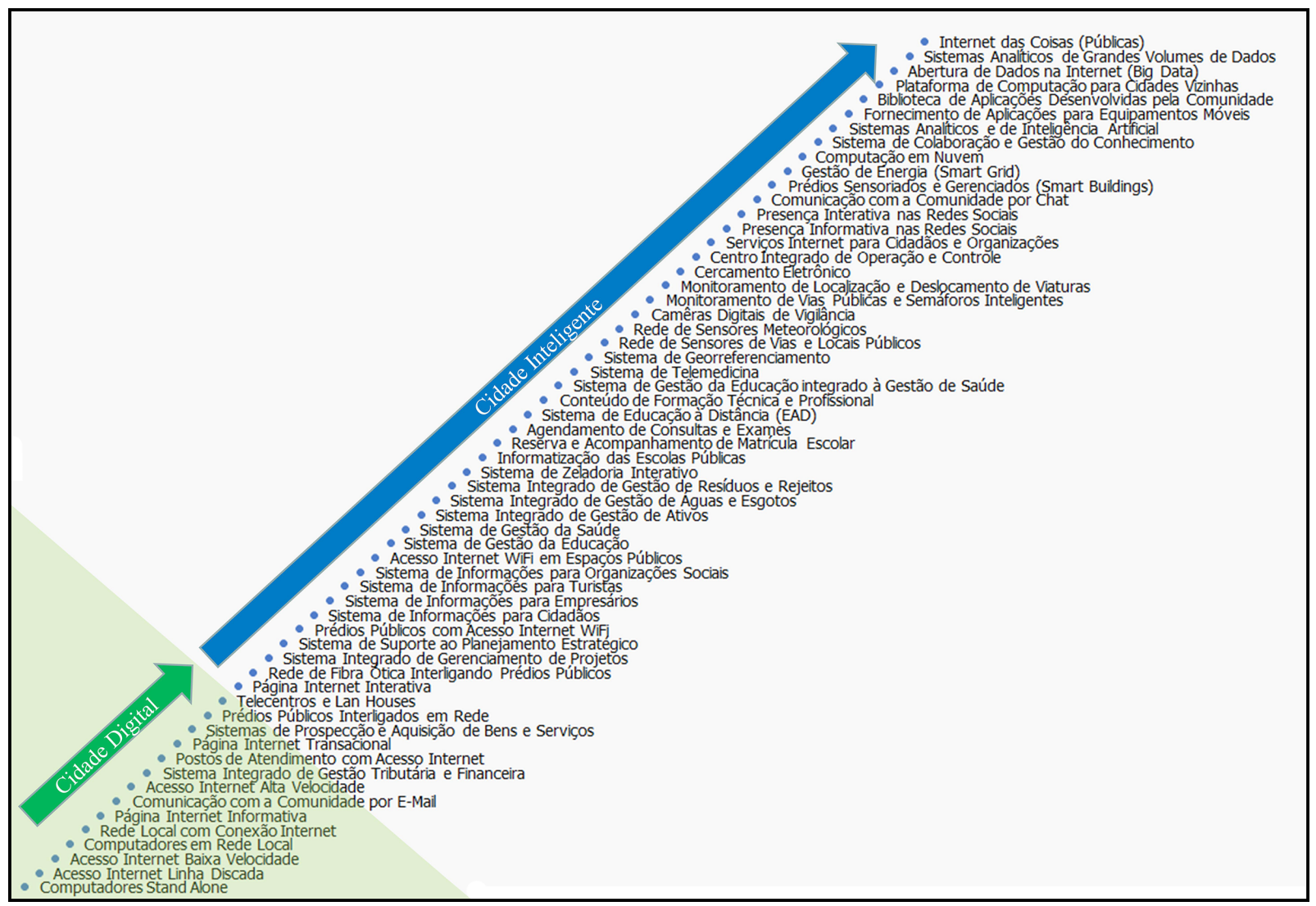

Figura 1 - Da cidade digital à cidade inteligente: caracterização da evolução Fonte: Elaborado pelos autores (2014).

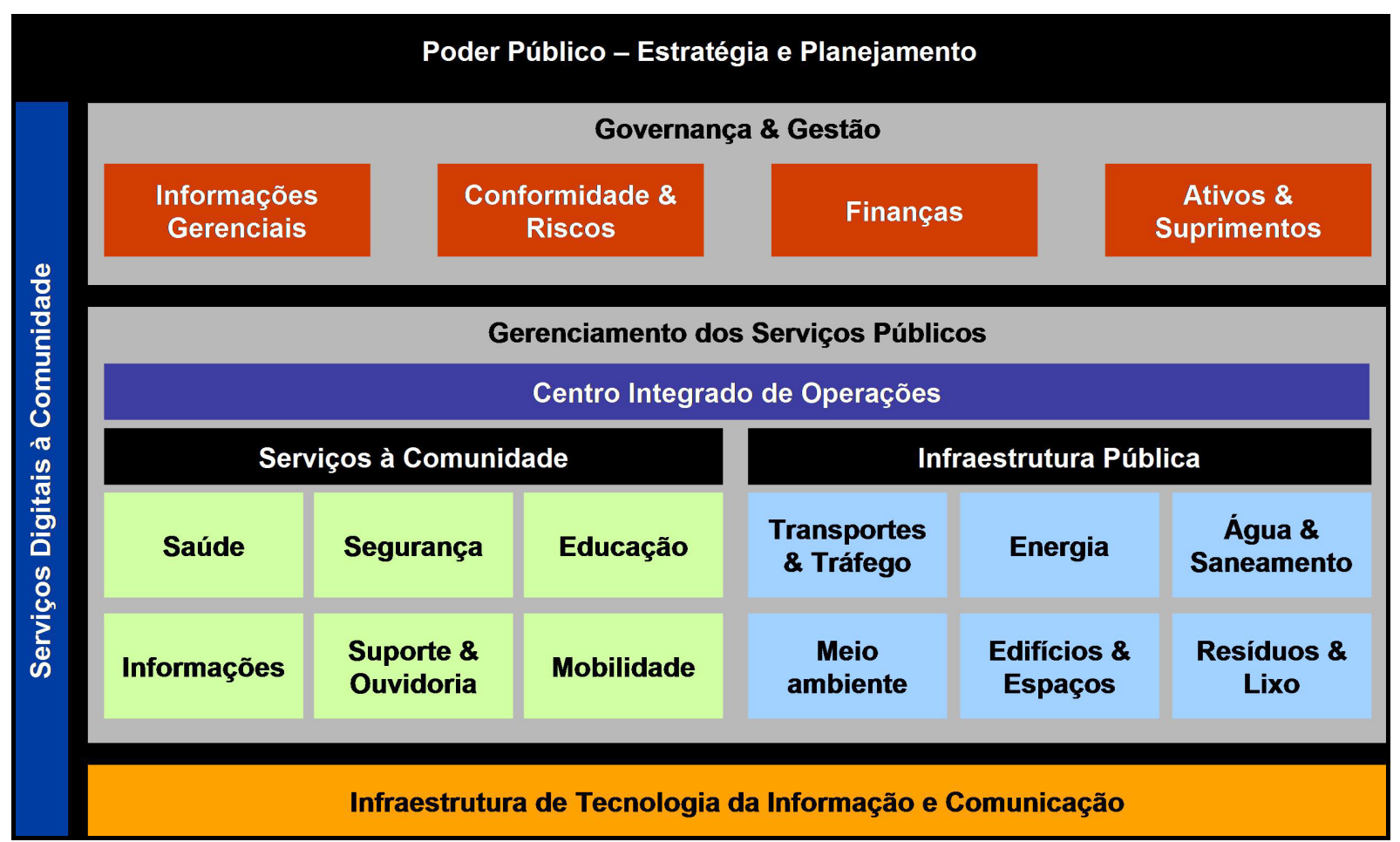

Figura 2 - Arquitetura de sistemas de gerenciamento para cidades inteligentes Fonte: Weiss (2013). 
uma evolução, de desenvolvimento socioeconômico e de um fenômeno global (Nam \& Pardo, 2011b) em que se busca a harmonização entre o mundo material e o mundo virtual, entre todos os subsistemas do sistema urbano, no melhor interesse dos atores que atuam nas cidades e respeitando suas características e vocações particulares (Boyko et al., 2006; Nam \& Pardo, 2011a; Toppeta, 2010; Rasoolimanesh et al., 2011).

\section{Procedimentos metodológicos}

Para este trabalho, foi realizada uma pesquisa qualitativa, exploratória, baseada em estudo de caso, permitindo "[...] uma investigação empírica que investiga um fenômeno contemporâneo dentro de seu contexto da vida real [...]" (Yin, 2005, p. 32).

Cinco critérios nortearam a escolha do objeto: 1) cidades brasileiras 2) com população acima de 1 milhão de habitantes, 3) diversidade de características geográficas, socioeconômicas e culturais, 4) apresentação no cenário brasileiro como cidade inteligente, e 5) apresentação no cenário internacional como cidade inteligente. Optou-se por realizar a pesquisa em Porto Alegre (POA) tendo em vista a disponibilidade dos agentes públicos em atender aos pesquisadores de forma célere e colaborativa.

Foram utilizadas fontes primárias e secundárias. As fontes primárias consistiram em entrevistas presenciais, semiestruturadas, seguindo um roteiro pré-elaborado, com gravação em áudio. Os resultados foram processados após cada entrevista de forma conjunta com a pesquisa bibliográfica e, posteriormente, com a análise documental. Este procedimento viabilizou a técnica de triangulação, possibilitando o exame mais consistente das evidências coletadas (Flick, 2004; Yin, 2005; Collis \& Hussey, 2006).

A escolha dos respondentes teve como direcionador a identificação de "[...] pessoas articuladas cultural e sensitivamente com o grupo ou organização [...]" (Gil, 2010, p. 121). Foram escolhidos agentes do poder público, titulares ou seus delegados, com autoridade para discorrer sobre a iniciativa de cidade inteligente em POA. As entrevistas foram realizadas no primeiro semestre de 2013, envolvendo representantes do poder público. Foram entrevistados: a Coordenadora de Relações Internacionais da Secretaria de Governança Local; o Coordenador do Escritório-Geral de Planejamento Estratégico da Secretaria de Planejamento Estratégico e Orçamento; o Assessor e Gerente de Projeto do Gabinete de Inovação (Inovapoa); o Supervisor da Secretaria de Meio Ambiente e o Coordenador de Relações Institucionais da Companhia de Processamento de Dados (Procempa).

Por meio das entrevistas, buscou-se identificar o alinhamento de conceitos entre os respondentes e o referencial teórico; motivações para a implementação da cidade inteligente; planos, resultados atuais e expectativas de resultados futuros, permitindo a avaliação de três dimensões particulares, como demonstrado na Figura 3.

Como fontes secundárias, foram utilizados documentos disponibilizados no portal da internet da prefeitura de POA, incluindo planos de governo, planos estratégicos, anuários estatísticos e relatórios de prestação de contas, além de notas jornalísticas.

A redação dos resultados da pesquisa foi estruturada de forma a caracterizar o ambiente de pesquisa e descrever a experiência da cidade relativamente à materialização do conceito de cidade inteligente. Tal procedimento possibilitou melhor descrição dos resultados, evitando-se perdas ou omissões de informações relevantes à conclusão do trabalho.

Por tratar-se de estudo de caso único, é relevante explicitar algumas limitações da pesquisa realizada. Os dados coletados basearam-se nas interações com os representantes do poder público e na consulta a documentos oficiais disponibilizados também pelo poder público, não permitindo o confronto de visões, opiniões e julgamentos dos diferentes atores que atuam na cidade. Outra limitação a ser considerada diz respeito à impossibilidade de generalização do caso para todas as cidades brasileiras, tendo em vista que o trabalho discute a aplicação do conceito em um local geográfico específico que não representa um padrão único para as demais cidades brasileiras. Entretanto, o método desenvolvido para a condução desta pesquisa poderá ser aplicado em outras localidades.

\section{Discussão dos resultados da pesquisa}

Porto Alegre (POA), fundada em 1772, tem área de $500 \mathrm{~km}^{2}$ e 1,4 milhão de habitantes aproximadamente, respondendo por um Produto Interno Bruto (PIB) da ordem de R 75 bilhões/ano (IBGE, 2012). 0 Índice de Desenvolvimento Humano (IDH) de POA, em 2013, foi de 0,805 , considerado muito alto, de acordo com 


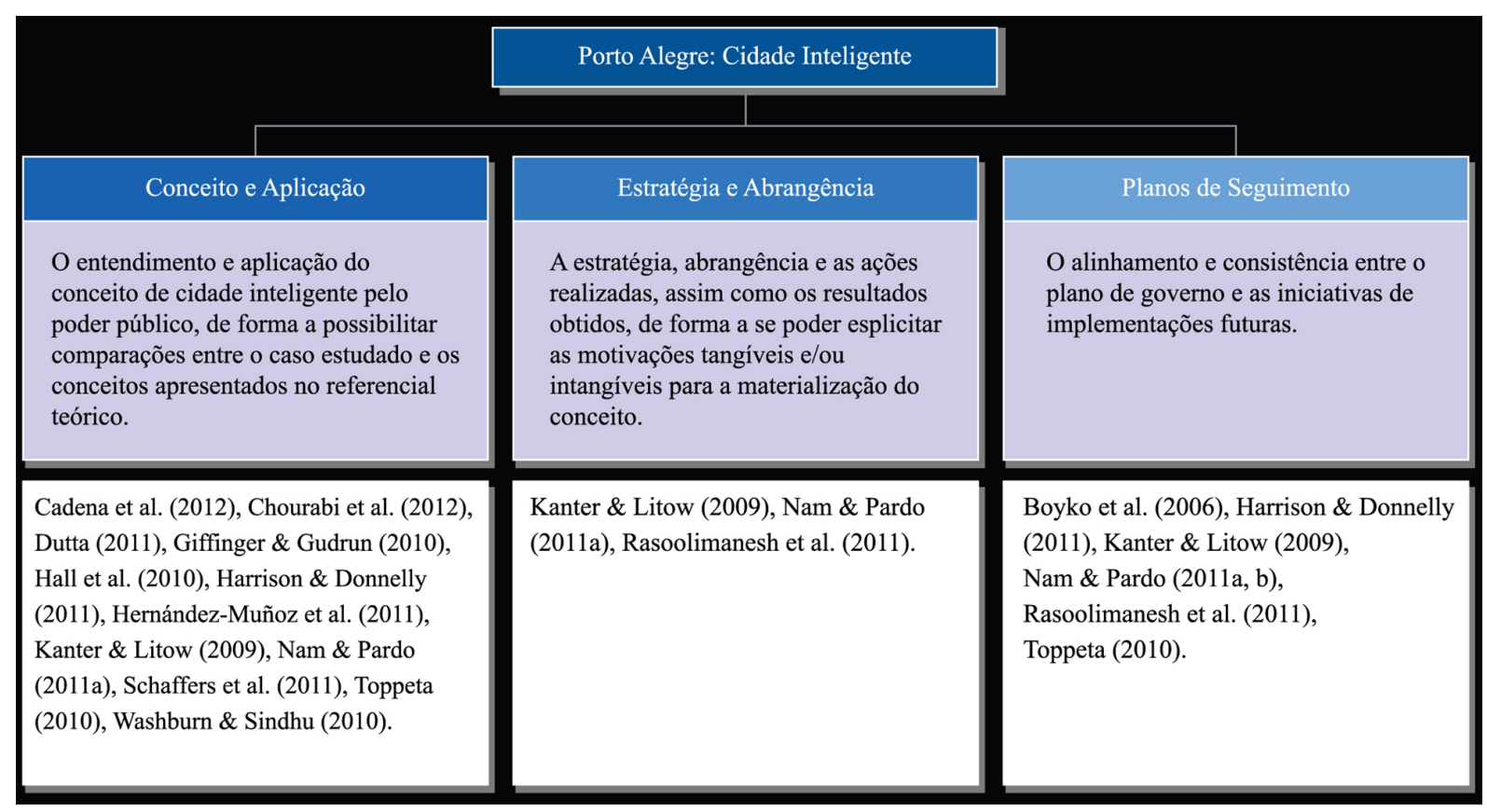

Figura 3 - Categorias de análise versus principais autores

Fonte: Elaborado pelos autores (2013).

os critérios do Programa das Nações Unidas para o Desenvolvimento (PNUD, 2010), e a expectativa de vida da população é de 72 anos.

\section{Origem e evolução das iniciativas} de TICs em Porto Alegre ${ }^{1}$

Desde 2006, POA tem implementado uma rede de fibras óticas que, em 2013, se estendia por aproximadamente $900 \mathrm{~km}$, garantindo acesso de alta velocidade à internet (155 Mbps) e conectando mais de 12 mil computadores nos diversos órgãos públicos, incluindo escolas da rede municipal e postos de saúde, oferecendo disponibilidade de 99,8\%. Com investimentos na ordem de R\$ 4 milhões até 2013, essa Infovia, primeira no Brasil, tem viabilizado a implantação de inovações em diferentes áreas de interesse dos atores, como saúde, educação e, segurança, entre outras.

No que diz respeito à sua presença na internet, o portal da prefeitura disponibiliza informações gerais que incluem ações do poder público e temas de interesse específico para estudantes, turistas, cidadãos e empreendedores. Pelo portal é possível

\footnotetext{
${ }^{1}$ Informações quantitativas representam posição em $31 / 12 / 2013$.
}

realizar transações tributárias, acionar a zeladoria da cidade ou concretizar procedimentos administrativos, como alvarás e licenças.

As ações de governo - das ações de execução do plano estratégico até as atividades das secretarias, órgãos e empresas públicas - estão divulgadas no portal da prefeitura (Prefeitura Municipal, 2013a), nos portais da Transparência (Prefeitura Municipal, 2013b), ObservaPOA (2013), de Gestão e Orçamento Participativo (Prefeitura Municipal, 2013c), além das páginas de cada órgão de governo que podem ser acessadas a partir da página principal da prefeitura. 0 volume de acessos a esses portais está representado no Quadro 2.

As redes sociais também integram o conjunto de instrumentos de relacionamento. A cidade conta com página no Facebook, da qual se pode acessar páginas relacionadas às ações do poder público, campanhas para conservação da cidade, agenda de eventos e ações de mobilização dos cidadãos em torno de alguma causa que julguem relevante, como apresentado no Quadro 3.

Na área de educação, o projeto Wireless Educação, inaugurado em 2007 e com investimentos de $\mathrm{R} \$ 2$ milhões, contempla a instalação de conexão sem fio de alta velocidade à internet na rede de ensino, promovendo a inserção dos alunos no mundo digital. 0 projeto permitiu a criação de um sistema para 
a rematrícula dos alunos do ciclo médio de forma online, que também podem acessar informações sobre seus respectivos históricos escolares. 0 projeto também incrementou a eficiência do processo administrativo da educação, reduzindo custos da ordem de R $\$ 1$ milhão/ano, anteriormente gastos com contratações descentralizadas de telecomunicações. As escolas possuem computadores modernos, idênticos aos utilizados nos órgãos de governo, permitindo que os alunos possam realizar seus acessos à internet $\mathrm{e}$ conteúdos educacionais sem restrições de capacidade e desempenho.

Em relação ao sistema público de saúde, merecem destaque três inovações. O Sistema de Telemedicina, criado em 2008, possibilita às gestantes a realização de exames de ultrassonografias nos postos de saúde. Trata-se de uma unidade móvel em que a paciente é examinada pelo médico no posto de saúde e o mesmo exame é acompanhado, em tempo real, por médico especialista no Hospital-Materno Infantil Presidente Vargas. Esta inovação reduziu as taxas de absentismo de $40 \%$ para $10 \%$, e o tempo de espera para a realização dos exames de quatro meses para um mês, fato que trouxe maior conforto e qualidade

Quadro 2 - Volume de acessos aos portais (em quantidade de acessos)

\begin{tabular}{ccrrr}
\hline Portal & $\mathbf{2 0 1 0}$ & \multicolumn{1}{c}{$\mathbf{2 0 1 1}$} & \multicolumn{1}{c}{$\mathbf{2 0 1 2}$} & \multicolumn{1}{c}{$\mathbf{2 0 1 3}$} \\
\hline Prefeitura & 8.543 .088 & 8.466 .155 & 9.628 .276 & 10.923 .935 \\
Transparência & 152.035 & 253.001 & 3.056 .944 & 2.527 .981 \\
ObservaPOA & 0 & 7.844 & 110.448 & 85.737 \\
Gestão & 53.624 & 54.820 & 133.536 & 139.204 \\
\hline
\end{tabular}

Fonte: Elaborado pelos autores (2013) com base nas entrevistas. de vida para as gestantes e proporcionou aumento de cerca de oito vezes na capacidade de realização de exames de atenção às mulheres gestantes.

A segunda, conhecida como Wireless Saúde, disponível desde 2011, é um sistema de informações integrado que permite que os usuários do Sistema Único de Saúde (SUS) sejam identificados unicamente e disponham de um prontuário eletrônico, acessível pelos agentes em qualquer ponto de atendimento, implementando o conceito de assistência continuada. O sistema contempla a implementação de uma base única de cadastro de usuário, profissionais e estabelecimentos. A rede de farmácias distritais, integrada à Infovia, também está no sistema integrado.

A terceira é a Estação Digital. Inaugurada em 2007, é uma parceria entre a Santa Casa de Misericórdia e a prefeitura, onde são disponibilizados computadores com acesso gratuito à internet aos pacientes $\mathrm{e}$ acompanhantes nos hospitais Santa Rita e da Criança Santo Antônio, resultando em aproximadamente 15 mil acessos mensais. São espaços equipados e que contam com voluntários treinados para auxiliar as pessoas no uso das tecnologias, promovendo a inclusão digital dos pacientes por meio da integração em grupo, atividades lúdicas, jogos pedagógicos que incentivam a aprendizagem, introdução à edição de textos, internet e comunicação através de webmails com amigos e familiares.

POA também dispõe de um Centro Integrado de Comando (CEIC). Inaugurado no final de 2012, recebeu investimentos na ordem de $\mathrm{R} \$ 5,6$ milhões e é o polo de monitoramento e operação da cidade. Está equipado com um painel de $48 \mathrm{~m}^{2}$, composto de 39 telas de 55 polegadas cada uma e 24 posições de trabalho, que podem reproduzir e controlar de forma particular qualquer evento apresentado no grande

Quadro 3 - Comunicação com os atores por meio de redes sociais

\begin{tabular}{|c|c|c|c|}
\hline Iniciativa nas redes sociais & Início & Curtidas & Descrição \\
\hline Porto Alegre: Eu curto. Eu cuido (2013) & 2010 & 2,6 mil & $\begin{array}{l}\text { Ambiente digital para promover atitudes construtivas com vista à construção de uma cidade } \\
\text { melhor. }\end{array}$ \\
\hline Nossa Porto Alegre (2013) & 2010 & 900 & $\begin{array}{l}\text { Espaço de comunicação sobre ações do poder público, campanhas de interesse geral e } \\
\text { interação entre os atores. }\end{array}$ \\
\hline Curtindo POA (2013) & 2012 & 13 mil & Agenda colaborativa criada pela prefeitura para divulgação de eventos de interesse público. \\
\hline Porto Alegre.cc (2013) & 2011 & 20 mil & $\begin{array}{l}\text { Plataforma digital onde se realizam discussões sobre a história, a realidade e o futuro de } \\
\text { territórios espećificos. }\end{array}$ \\
\hline
\end{tabular}

Fonte: Elaborado pelos autores (2013). 
painel. Essas posições são utilizadas durante 24 horas por dia, 7 dias por semana. São monitorados mais de 840 pontos, incluindo espaços e prédios públicos, de forma integrada e simultânea, em tempo real, com o auxílio de câmeras dotadas de cerca eletrônica e alarmes para mobilização de agentes de segurança. O CEIC integra diversos órgãos municipais, como mostrado na Figura 4, além dos parceiros dos demais poderes que utilizam as instalações quando necessário para o monitoramento de eventos e ocorrências de suas competências. Conta também com uma sala para a imprensa, disponível 24 horas por dia, de onde se podem acompanhar temas de interesse da população.

Além do sistema de monitoramento, o CEIC recebe informações provenientes de radares meteorológicos; sensores de nível de rios; monitoramento do fluxo de veículos, incluindo transporte coletivo, nas principais vias da cidade; localização e gerenciamento de viaturas, com suporte remoto em caso de necessidade de primeiros socorros; facilidades de telecomunicações e outras funcionalidades. Com o início das operações do CEIC, POA começa a fornecer serviços interoperáveis que facilitam a integração e transformação de processos de governo, internamente entre as agências e departamentos e, externamente, para cidadãos e empresas.

Com relação à energia, à água e ao saneamento, aos edifícios e espaços, e à zeladoria pública, as tecnologias estão sendo contempladas com ações de modernização. A criação de um novo sistema de gerenciamento de ativos com sensores integrados, implantado entre 2012 e 2013, tem proporcionado

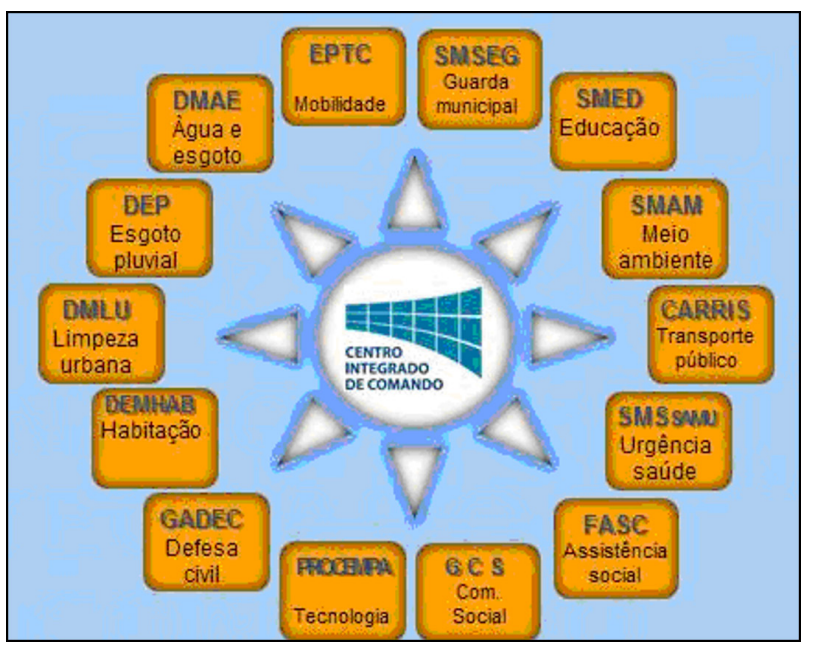

Figura 4 - Agências presentes no CEIC Fonte: Prefeitura Municipal (2013a). agilidade no atendimento às necessidades de manutenções das infraestruturas públicas, melhor controle no uso de materiais e suprimentos e eficiência da força de trabalho. 0 sistema permite que os cidadãos interajam com o poder público, registrando e acompanhando suas demandas relativamente aos serviços públicos (água, esgoto, vias públicas, limpeza urbana etc.). Os departamentos contemplados com a solução podem gerenciar obras e reparos que estão sendo feitos na cidade, administrando de forma efetiva a utilização de materiais e o ciclo de vida dos ativos públicos.

Ainda no campo da gestão das infraestruturas e serviços públicos, POA tem um sistema de geoprocessamento, funcionando como uma fotografia da cidade. Nele são destacados os postos de oferta de serviços públicos, redes de esgoto, praças, locais de eventos e outros pontos de interesse. É utilizado para apoiar várias iniciativas, como o portal ObservaPOA, Plano Diretor de Drenagem, Copa 2014, Plano Cicloviário, Projeto Integrado Entrada da Cidade, Zoneamento Ambiental, Combate à Dengue, Programa Reluz e Projeto Integrado Socioambiental. Neste campo, o portal ObservaPOA merece destaque. A iniciativa, desenvolvida por meio de parceria entre a prefeitura e a Universidade Federal do Rio Grande do Sul (UFRGS), estabelecida em 2007, possibilita a disponibilização e visualização georreferenciada dos indicadores socioeconômicos da cidade. 0 georreferenciamento é feito por regiões e bairros, reforçando a identidade do local e promovendo o aprofundamento dos estudos sobre os acontecimentos em cada região da cidade. 0 portal disponibiliza indicadores para apoiar a gestão participativa seguindo três perspectivas: social, gestão e política.

A inclusão digital também é um aspecto contemplado na Porto Alegre mais Inteligente.

A Procempa Livre e Gratuita, cuja implementação se iniciou em 2006, é uma rede pública para acesso sem fio à internet, com infraestrutura de rádios ligados à Infovia. $\mathrm{O}$ acesso está disponibilizado em todos os prédios públicos, praças e parques, e também, sob demanda, nos locais destinados a eventos, totalizando 47 lugares públicos contemplados com 112 rádios instalados. A rede registrou mais de 500 milhões de acessos a sites na internet no ano de 2013 e tem se constituído em importante iniciativa para a inclusão digital na cidade.

Outra iniciativa é o PortoWeb, provedor de internet mantido pelo poder público e que disponibiliza 
serviços de acesso, correio eletrônico, desenvolvimento e hospedagem de sites para a prefeitura e para a comunidade. 0 PortoWeb também é responsável pelo Portal da Solidariedade, desenvolvendo e hospedando páginas para organizações não governamentais e entidades sem fins lucrativos. 0 provedor encerrou o ano de 2013 com mais de 4 milhões de acessos e 1.180 sites hospedados.

Ainda no âmbito da inclusão digital, a Escola de Desenvolvedores de Software é uma parceria entre a Procempa, Associação das Empresas Brasileiras de Tecnologia da Informação - Regional RS (Assespro/RS) e Secretaria Municipal de Educação (SMED), que tem por objetivo capacitar os jovens da rede pública de ensino em tecnologia da informação.

A cidade também tem espaços para capacitação digital da população. Criados a partir de 2004, os Centros de Capacitação Digital (CCD) oferecem cursos de introdução à informática, sistema, internet, e-mail, redes sociais, editores de textos e de apresentações e planilhas eletrônicas, todos apoiados por apostilas e cadernos de exercícios. A iniciativa reúne 8 centros, 12 salas de aulas, aproximadamente 200 computadores em rede e, desde seu surgimento, já capacitou mais de 34 mil cidadãos.

A prefeitura, por intermédio da Procempa, disponibiliza acesso gratuito à internet, transmissão de vídeo e recursos de videomonitoramento para eventos culturais e de entretenimento, tais como: Carnaval, Fórum Internacional de Software Livre, Acampamento Farroupilha, Congresso Internacional de Gestão, Expointer, Bienal do Mercosul, Semana Municipal de Ciência e Tecnologia, Regional Porto Alegre Robotics, Feira do Livro de Porto Alegre e Salão Náutico do Mercosul.

\section{Ações futuras: perspectivas para ampliação do uso das TICs}

Os planos de seguimento englobavam novos sistemas para os serviços de saúde, com o aprimoramento do prontuário único do paciente e outras facilidades para os agentes e cidadãos, incluindo o agendamento eletrônico de consultas para $70 \%$ da rede e o sistema de regulação de leitos, ambos em 2013.

Relativamente à segurança pública, para o ano de 2013, no âmbito das competências do município, estavam a cobertura de $100 \%$ de comunicação móvel para serviços críticos; a integração de $100 \%$ das bases de dados ao CEIC e a inclusão de mais 600 câmeras de monitoramento; maior inserção da Guarda Municipal no CEIC, além de operações conjuntas com a Brigada Militar.

Nos planos para 2013 também estavam a conclusão da implantação do Sistema Integrado de Administração Tributária; a implementação de $80 \%$ do processo digital da análise de grandes empreendimentos e 100\% do projeto de emissão de Declaração Municipal (DM), ambos no âmbito do processo de Licenciamento e Regularização Fundiária.

A cidade também planejava a atração de mais 30 novas empresas do segmento de TICs, a ampliação de 647 para 712 de bolsas universitárias no projeto UNIPOA, a instalação do banco de ideias e projetos e a criação de um polo de economia criativa, todos em 2013.

Sobre a implantação de um polo de economia criativa, a cidade planejava realizar o primeiro projeto até o final de 2013 e tinha como objetivos o incremento do desenvolvimento local de forma sustentável e a alavancagem de ideias e empreendimentos, por meio da incubação de empresas, formação e capacitação dos atores dos arranjos produtivos locais, proporcionando maior proximidade entre o poder público, a iniciativa privada, a academia e a sociedade.

Além dessas significativas ações, outras que visavam à otimização da prestação de serviços estavam planejadas para 2013, como a adoção de equipamentos de GPS em $100 \%$ da frota de táxis, novos sistemas e funcionalidades para a redução dos tempos de atendimento e trâmite de processos e demandas dos atores, melhoria do processo de gestão de recursos humanos por meio do sistema de ponto eletrônico e outras.

Porto Alegre: uma cidade inteligente? Uma análise dos resultados

Ao se iniciar a análise dos resultados, adota-se o conceito de cidade inteligente postulado por Steinert et al. (2011, p. 87), para quem

[...] as cidades inteligentes têm foco em um modelo particularizado, com visão moderna do desenvolvimento urbano e que reconhecem a crescente importância das TICs no direcionamento da competitividade econômica, sustentabilidade ambiental e qualidade de vida geral; esse 
conceito vai além dos aspectos puramente técnicos que caracterizam as cidades como cidades digitais [...].

POA tem materializado o conceito de cidade inteligente por meio da implementação de TICs de acordo com boas práticas de mercado, utilizando planos estratégicos e gestão por objetivos, desenvolvendo e adotando novas formas de execução de seus processos internos, interação e cooperação entre os agentes de diferentes órgãos, a partir do compartilhamento de informações. Alinhando-se ao arcabouço teórico explorado neste trabalho, POA reconhece que a cidade inteligente é aquela que faz uso extensivo das TICs em benefício dos atores.

O plano de governo considera o uso das TICs como meio para incrementar suas práticas de governança, inovação e desenvolvimento sustentável; estreitar a comunicação com os atores; promover o bem-estar das pessoas e pavimentar o caminho das gerações futuras em direção a um ambiente urbano mais favorável.

A implementação das TICs está ocorrendo com foco nas necessidades dos atores. As infraestruturas de TICs estão sendo construídas de forma a possibilitar a integração entre as pessoas, as empresas e o governo, em âmbitos regional, nacional e, futuramente, internacional. Os sistemas de informações, a despeito das melhorias e avanços ainda necessários, buscam aumentar a eficiência dos serviços públicos, contemplando as diferentes facetas da cidade: planejamento, desenvolvimento, implantação e manutenção. Com as tecnologias instaladas, $\mathrm{o}$ aparelhamento dos pontos de atendimento ao público tem sido de importância para a melhoria da qualidade de vida dos cidadãos, principalmente para aqueles que dependem exclusivamente dos serviços públicos.

Quanto ao conceito de desenvolvimento sustentável, a cidade tem buscado se posicionar como referência. Graças ao Modelo de Gestão e ao Orçamento Participativo, POA tem estabelecido ações que visam ao equilíbrio financeiro, à proteção ambiental e à justiça social, como descrito em seus programas estratégicos. Esses programas contam com as TICs como importantes viabilizadoras, tanto no momento em que ainda se configuram como planos, quanto no momento em que seus resultados são entregues à população e, portanto, deverão ser mantidos.

Os avanços pretendidos apontam para a continuidade dos investimentos em TICs, de forma alinhada ao Orçamento Participativo, visando ao aprimoramento e à complementação do que já foi implementado.
A análise dos dados sugere que as TICs têm funcionado como um sistema nervoso em POA, ativando ramificações que atingem todos os atores que lá atuam. É também por meio dessas tecnologias que o poder público tem fomentado e realizado esforços com vistas ao aprimoramento da colaboração entre os atores, visando à construção de uma cidade mais criativa, inclusiva, sustentável e inteligente.

Os investimentos realizados na implementação de inovações em infraestruturas tecnológicas e sistemas de informações vão colocando POA na trilha das cidades inteligentes.

Muitas são as ações notadas na cidade. Entretanto, percebe-se que há oportunidades de melhorias para benefício de todos os atores. Algumas dessas melhorias envolvem: exploração mais adequada do portal da prefeitura na internet, com gerenciamento de conteúdo; acesso mais amigável a links e opção de outros idiomas; novas funcionalidades no portal para a realização de transações e solicitações de serviços, evitando o deslocamento dos atores aos postos de atendimento; ampliação da abrangência do sistema de gestão de ativos; ampliação das funcionalidades e abrangência da solução integrada de gestão da saúde, educação e ações sociais, permitindo melhor acompanhamento do uso desses serviços por meio do correlacionamento de informações, agilidade na prestação de serviços e melhoria nas capacidades de informações para a tomada de decisões; ampliação sistemática de sensores nos ativos de transportes públicos - ônibus, táxis - propiciando melhor monitoramento e planejamento de ações de mobilidade na cidade.

Além dessas possibilidades, o poder público pode aprimorar as capacidades do CEIC com sistemas analíticos que façam uso do grande volume de dados (big data) gerado pelos sensores, agências, mídias sociais e atores; promover a abertura de dados, permitindo que quaisquer atores, particularmente aqueles voltados à construção de aplicativos para dispositivos móveis, possam ter acesso e propor inovações que beneficiem a cidade como um todo. Implementar tecnologias no gerenciamento dos prédios públicos, com vistas ao uso mais racional dos espaços, energia, água e infraestrutura predial; incentivar a implementação de programas e tecnologias de smart grid e programas de eficiência energética; captação, reaproveitamento e eficiência no fornecimento de água, particularmente nos espaços públicos, são iniciativas que também merecem atenção do poder público. 


\section{Considerações finais}

Este artigo traz uma reflexão sobre questões ligadas ao fenômeno da urbanização, seus desafios e oportunidades. Refletindo sobre estas questões, explorou-se o conceito de cidade inteligente e as possibilidades de sua materialização sob a perspectiva das TICs, de forma a permitir que governos, iniciativa privada, organizações não governamentais, academia e cidadãos possam enfrentar e resolver os intrincados problemas organizacionais, sociais e materiais advindos da rápida e intensa urbanização. Para ilustrar essa reflexão, este artigo focalizou a experiência particular de POA em seus esforços para se transformar em uma cidade inteligente.

Não obstante a impossibilidade de generalização para outras cidades brasileiras, POA se projeta no cenário nacional como potencial fonte de conhecimento sobre esse tipo de iniciativa.

A exploração do caso de POA sugere que os desafios de se implementar cidades inteligentes no Brasil não são pequenos, particularmente quando graves questões sociais ainda carecem de melhor enfrentamento: renda, saneamento básico, educação, mobilidade, saúde e segurança. Tais questões, entretanto, podem ser encaradas como motivadores para que governos, empresas e academia unam esforços para criar cidades brasileiras mais inteligentes e sustentáveis.

Há que se considerar, entretanto, que a proposição de cidades inteligentes deve ser vista e avaliada com cautela. 0 discurso da cidade inteligente não deve retratar um local imaginário ou utópico, para onde convergem todos as ideias de desenvolvimento sustentável e de democratização do acesso e bom uso da informação. Ao contrário, deve apontar para uma forma pragmática e factível sobre como tais ideias podem ser materializadas.

Se, por um lado, o discurso pode ser utilizado para que se desenvolvam projetos criteriosos para a melhoria das condições de vida nas cidades, por outro ele pode ser usado por gestores públicos ou por agremiações políticas para a manutenção de agendas que não necessariamente passam pelo bem-estar da sociedade e pelo respeito aos princípios da boa governança: ética, transparência, equidade, prestação de contas, conformidade e responsabilidade objetiva.

Além disso, o discurso das cidades inteligentes - facilitador para a melhoria da vida nas cidades também tem sido apropriado de forma significativa por muitas empresas da indústria de TICs. Para além da contribuição que estas empresas oferecem em termos de avanços tecnológicos, há que se considerar sua obrigação para a realização de negócios.

A implementação de componentes de TICs não dever ser vista como a solução única e inequívoca para a resolução dos problemas nas infraestruturas e serviços públicos que muitas cidades têm experimentado. É imperativo que sua implementação seja respaldada por criteriosas avaliações de aplicabilidade e de benefícios auferidos como consequência dos investimentos realizados. Decidir cuidadosamente sobre a estratégia a ser seguida, particularmente considerando aspectos financeiros, legais e políticos, é de especial importância. Esses aspectos, entretanto, não podem ser encarados como restrições intransponíveis: acordos de colaboração, convênios, parcerias público-privadas e outras formas de cooperação podem ser utilizadas na melhor forma do direito e no melhor interesse das cidades.

Pretendeu-se com este trabalho contribuir com a arena de discussões sobre como o uso das TICs pode aprimorar o gerenciamento dos serviços públicos, estimular a iniciativa privada a ações que visem à melhoria da qualidade de vida nos centros urbanos e à maior interação com o poder público, e a academia com vistas a soluções para os problemas decorrentes da intensa urbanização nas cidades brasileiras.

Futuros estudos podem contemplar estudos comparativos entre cidades brasileiras; avaliação do impacto de aspectos e/ou componentes específicos das TICs para o fornecimento de serviços públicos; avaliação do uso e impacto das redes sociais no relacionamento entre o poder público e os atores da cidade; estudos sobre a viabilidade para a criação de regiões inteligentes; e novas proposições e modelos baseados em TICs para o gerenciamento dos serviços públicos.

\section{Referências}

Al-hader, M., \& Rodzi, A. (2009). The smart city infrastructure development \& monitoring. Theoretical and Empirical Researches in Urban Management, 4(2), 87-94.

Allwinkle, S., \& Cruickshank, P. (2011). Creating smart-er cities: an overview. Journal of Urban Technology, 18(2), 1-16. http://dx.doi.org/10.1080/10630732.2011.601103 
Batagan, L. (2011). Smart cities and sustainability models. Informatica Economica, 15(3), 80-87.

Bollier, D. (1998). How smart growth can stop sprawl: a fledgling citizen movement expands. Washington: Essential Books.

Boyko, C. T., Cooper, R., Davey, C. L., \& Wootton, A. B. (2006). Addressing sustainability early in the urban design process. Management of Environmental Quality: an International Journal, 17(6), 689-706.

Brenner, N. (1998). Global cities, glocal states: global city formation and state territorial restructuring in contemporary Europe. Review of International Political Economy, 5(1), 1-37. http://dx.doi.org/10.1080/096922998347633

Cadena, A., Dobbs, R., \& Remes, J. (2012). The growing economic power of cities. Journal of International Affairs, 65(2), 1-17.

Castells, M. (2012). A sociedade em rede (6a ed.) São Paulo: Paz e Terra.

Chourabi, H., Nam, T., Walker, S., Gil-Garcia, J. R., Mellouli, S., Nahon, K., Pardo, T., \& Scholl, H. J. (2012). Understanding smart cities: an integrative framework. In Anais do XLV Hawaii International Conference on System Sciences (p. 2289-2297). Albany: Center for Technology in Government.

Coelho, F. D. (2010). Desenvolvimento local e sociedade da informação. In L. Dowbor, \& M. Pochmann (Orgs.), Políticas para o desenvolvimento local (p. 337-365). São Paulo: Fundação Perseu Abramo.

Cohen, M. P. (2011). Cities in times of crisis: the response of local government in light of the global economic crisis - the role of the formation of humam capital, urban innovation and strategic planning. Berkeley: Institute of Urban and Regional Development. Recuperado em 28 de janeiro de 2013, de https://escolarship.org/uc/item/3432p4rb

Collis, J., \& Hussey, R. (2006). Pesquisa em administração: um guia prático para alunos de graduação e pós-graduação (2a ed). Porto Alegre: Bookman.

Curtindo POA. (2013). Recuperado em 31 de dezembro de 2013, de https://www.facebook.com/CurtindoPOA?fref=ts

Dirks, S., Gurdgiev, C., \& Keeling, M. (2010). Smarter cities for smarter growth: how cities can optimize their systems for the talent-based economy (p. 1-14, Executive Report). Somers: IBM Institute for Business Value. Recuperado em 21 de setembro de 2012, de http://ssrn.com/abstract=2001907
Dodgson, M., \& Gann, D. (2011). Technological innovation and complex systems in cities. Journal of Urban Technology, 18(3), 101-113. http://dx.doi.org/10.1080/10630732.2 011.615570

Dutta, S. (Ed.). (2011). The Global Innovation Index 2011: accelerating growth and development. Fontainebleau: INSEAD.

Eger, J. M. (2009). Smart growth, smart cities, and the crisis at the pump a worldwide phenomenon. I-Ways, 32(1), 47-53.

Flick, U. (2004). Uma introdução à pesquisa qualitativa (2a ed.). Porto Alegre: Bookman.

Friedmann, J. (1986). The world city hypothesis. Development and Change, 17(1), 69-83. http://dx.doi. org/10.1111/j.1467-7660.1986.tb00231.x

Giffinger, R., \& Gudrun, H. (2010). Smarter cities ranking: an effective instrument for the positioning of cities? ACE: Architecture, City and Environment, 12, 7-25.

Gil, A. C. (2010). Como elaborar projetos de pesquisa (5a ed). São Paulo: Atlas.

Gupta, J. (2002). Global sustainable development governance: institutional challenges from a theoretical perspective. International Environmental Agreement: Politics, Law and Economics, 2(4), 361-388. http://dx.doi. org/10.1023/A:1021387308065

Hall, R. E., Bowerman, B., Braverman, J., Taylor, J., Todosow, H., \& von Wimmersperg, U. (2000). The vision of a smart city. In Proceedings of the 2nd International Life Extension Technology Workshop (p.1-6). Upton: Brookhaven National Laboratory. Recuperado em 15 de agosto de 2012, de http://www.osti.gov/bridge/servlets/purl/773961-oyxp82/ webviewable/773961.pdf

Hammer, S., Kamal-Chaoui, L., Robert, A., \& Plouin, M. (2011). Cities and green growth: a conceptual framework (OECD Regional Development Working Papers 2011/08). Paris: OECD Publishing. Recuperado em 04 de setembro de 2012, de http://dx.doi.org/10.1787/5kg0tflmzx34-en Harrison, C., \& Donnelly, I. A. (2011). A theory of smart cities. In Proceedings of the 55th Annual Meeting of the International Society for the Systems Science (p. 1-15). Hull: University of Hull. Recuperado em 09 de junho de 2012, de http://journals.isss.org/index.php/proceedings55th/ article/viewFile/1703/572

Hernández-Muñoz, J. M., Vercher, J. B., Muñoz, L., Galache, J. A., Presser, M., Hernández Gómez, L. A., \& Pettersson, J. 
(2011). Smart cities at the forefront of the future internet. Lecture Notes in Computer Science, 6656, 447-462. http:// dx.doi.org/10.1007/978-3-642-20898-0_32

Instituto Brasileiro de Geografia e Estatística - IBGE. (2012). Censo demográfico - 2010. Rio de Janeiro: IBGE. Recuperado em 10 de junho de 2012, de http://www. ibge.gov.br/home/estatistica/ populacao/censo2010/ default.shtm

Johnson, B. (2008). Cities, systems of innovation and economic development. Innovation: Management, Policy \& Practice, 10(2-3), 146-155. http://dx.doi.org/10.5172/ impp.453.10.2-3.146

Kanter, R. M., \& Litow, S. S. (2009). Informed and interconnected a manifesto for smarter cities. Harvard Business School General Management Unit Working Paper, 9(141), 1-27. Recuperado em 14 de agosto de 2012, de http://papers. ssrn.com/sol3/papers.cfm?abstract_id=1420236

Komninos, N. (2002). Intelligent cities: innovation, knowledge systems, and digital spaces. London: Spon Press.

Komninos, N., \& Sefertzi, E. (2009). Intelligent cities: R\&D offshoring, Web 2.0 product development and globalization of innovation systems. In Proceedings of the Second Knowledge Cities Summit 2009 (p. 1-8). Shenzhen: World Capital Institute. Recuperado em 06 de agosto de 2013, dehttp://www.urenio.org/wp-content/uploads/2008/11/ Intelligent-Cities-Shenzhen-2009-Komninos-Sefertzi.pdf

Komninos, N., Schaffers, H., \& Pallot, M. (2011). Developing a policy roadmap for smart cities and the future internet. In Echallenges e-2011 Conference Proceedings (p. 1-8). Thessalonik: URENIO - Urban and Regional Innovation Research. Recuperado em 14 de setembro de 2012, de http://www.urenio.org/wp-content/uploads/2008/11/2011eChallenges_ref_196-Roadmap-for-Smart-Cities-Publised.pdf

Mitchell, W. J. (2007). Intelligent cities. E-Journal on the Knowledge Society, (5), 3-8. Recuperado em 15 de junho de 2012, de http://www.uoc.edu/uocpapers/5/dt/eng/ mitchell.pdf

Nam, T., \& Pardo, T. A. (2011a). Conceptualizing smart city with dimensions of technology, people and institutions. In Proceedings of the 12th Annual International Conference on Digital Government Research (p. 282-291). New York: ACM. Recuperado em 10 de junho de 2012, de http://www.ctg. albany.edu/publications/journals/dgo_2011_smartcity/ dgo_2011_smartcity.pdf
Nam, T., \& Pardo, T. A. (2011b). Smart city as urban innovation: focusing on management, policy and context. In Proceedings of the 5th International Conference on Theory and Practice of Electronic Governance (p. 185-194). New York: ACM. Recuperado em 20 de junho de 2012, de http://www.ctg.albany.edu/publications/journals/ icegov_2011_smartcity

Nossa Porto Alegre. (2013). Recuperado em 31 de dezembro de 2013, de https://www.facebook.com/ NossaPortoAlegre/info

Observatório da Cidade de Porto Alegre - ObservaPOA. (2013). Porto Alegre: Prefeitura Municipal. Recuperado em 28 de abril de 2013, de http://www.observapoa.com.br/

Organização das Nações Unidas - ONU. (2012). World urbanization prospects: the 2011 revision. New York: ONU. Recuperado em 06 de junho de 2012, de http://esa.un.org/ unpd/wup/index.htm

Parkinson, M., Hutchins, M., Simmie, J., Clark, G., \& Verdonk, H. (2004). Competitive European Cities: where do the core cities stand? London: Her Majesty's Stationery Office. Recuperado em 20 de junho de 2012, de http://www. vrm.ca/documents/competitive.pdf

Pastor, M., Lester, T. W., \& Scoggins, J. (2009). Why regions? Why now? Who cares? Journal of Urban Affairs, 31(3), 269296. http://dx.doi.org/10.1111/j.1467-9906.2009.00460.x

Porto Alegre.cc. (2013). Recuperado em 31 de dezembro de 2013, de https://www.facebook.com/poa.cc?fref=ts

Porto Alegre: Eu curto. Eu cuido. (2013). Recuperado em 31 de dezembro de 2013, de https://www.facebook.com/ eucurtoeucuido

Prattipati, S. N. (2010). Sustainability and the role of information and communications technologies. Business Renaissance Quarterly, 5(2), 23-40.

Prefeitura Municipal. (2013a). Portal da Prefeitura de Porto Alegre. Porto Alegre: Prefeitura Municipal. Recuperado em 28 de abril de 2013, de http://www2.portoalegre.rs.gov. br/portal_pmpa_novo/

Prefeitura Municipal. (2013b). Portal transparência e acesso à informação. Porto Alegre: Prefeitura Municipal. Recuperado em 28 de abril de 2013, de http://www2. portoalegre.rs.gov.br/transparencia/

Prefeitura Municipal. (2013c). Orçamento participativo. Porto Alegre: Prefeitura Municipal. Recuperado em 28 
de abril de 2013, de http://www2.portoalegre.rs.gov.br/ op/default.php

Programa das Nações Unidas para o Desenvolvimento PNUD. (2010). Atlas do Desenvolvimento Humano no Brasil: ranking - todo o Brasil (2010). Recuperado em 28 de abril de 2013, de http://www.atlasbrasil.org.br/2013/pt/ranking

Rasoolimanesh, S. M., Badarulzaman, N., \& Jaafar, M. (2011). Achievement to sustainable urban development using city development strategies: a comparison between cities alliance and the World Bank definitions. Journal of Sustainable Development, 4(5), 151-166. http://dx.doi. org $/ 10.5539 /$ jsd.v4n5p151

Sassen, S. (1998). As cidades na economia mundial. São Paulo: Studio Nobel.

Sassen, S. (2005). The global city: introducing a concept. The Brown Journal of World Affairs, 11(2), 27-43.

Schaffers, H., Komninos, N., Pallot, M., Trousse, B., Nilsson, M., \& Oliveira, A. (2011). Smart cities and the future internet: towards cooperation frameworks for open innovation. Lecture Notes in Computer Science, 6656, 431-446.

Scott, A. J., Agnew, J., Soja, E. W., Storper, M. (2001). Global city-regions: an overview. In A. J. Scott (Ed.), Global cityregions: trends, theory, policy (p. 11-30). Oxford: Oxford University Press.

Steinert, K., Marom, R., Richard, P., Veiga, G., \& Witters, L. (2011). Making cities smart and sustainable. In S. Dutta (Ed.), The Global Innovation Index 2011: accelerating growth and development (p. 87-96). Fontainebleau: INSEAD

Storper, M. (1997). The city: centre of economic reflexivity. Service Industries Journal, 17(1), 1-27. http://dx.doi. org/10.1080/02642069700000001
Toppeta, D. (2010). The smart city vision: how innovation and ICT can build smart, "livable", sustainable cities. Milão: The Innovation Knowledge Foundation. Recuperado em 15 de junho de 2012, de http://www.thinkinnovation. org/file/research/23/en/Toppeta_Report_005_2010.pdf

Washburn, D., \& Sindhu, U. (2010). Helping CIOs understand "smart city" initiatives: defining the smart city, its drivers, and the role of the CIO. Cambridge: Forrester Research. Recuperado em 18 de junho de 2012, de http://www-935.ibm.com/ services/us/cio/pdf/forrester_help_cios_smart_city.pdf

Webber, L., \& Wallace, M. (2009). Green tech: how to plan and implement sustainable IT solutions. New York: AMACON.

Weiss, M. C. (2013). Cidades inteligentes como nova prática para o gerenciamento dos serviços e infraestruturas urbanos: estudo de caso da cidade de Porto Alegre (Dissertação de mestrado). Centro Universitário da FEI, São Paulo.

Wolfram, M. (2012). Deconstructing smart cities: an intertextual reading of concepts and practices for integrated urban and ICT development. In Proceedings of the REAL CORP 2012 Tagungsband (p. 171-181). Schwechat: Competence Center for Urban and Regional Planning.

Yin, R. K. (2005). Estudo de caso: planejamento e métodos (3a ed.). Porto Alegre: Bookman.

Yovanof, G. S., \& Hazapis, G. N. (2009). An architectural framework and enabling wireless technologies for digital cities and intelligent urban environments. Wireless Personal Communications, 49(3), 445-463. http://dx.doi.org/10.1007/ s11277-009-9693-4

Recebido: Set. 16, 2014

Aprovado: Dez. 15, 2014 